\title{
Direct Determination of Ground-State Transition Widths and Natural Level Widths with the Method of Relative Self Absorption
}

\author{
C. Romig ${ }^{1, a}$, T. Beck ${ }^{1}$, J. Beller ${ }^{1}$, J. Birkhan ${ }^{1}$, A. Endres ${ }^{2}$, M. Fritzsche ${ }^{1}$, U. Gayer ${ }^{1}$, J. Glorius ${ }^{2}$, J. Isaak ${ }^{3,4}$, L. Mertes ${ }^{1}$, \\ H. Pai ${ }^{1}$, N. Pietralla ${ }^{1}$, P. Ries ${ }^{1}$, D. Savran ${ }^{3,4}$, M. Scheck ${ }^{1,5,6}$, L. Schnorrenberger ${ }^{1}$, K. Sonnabend ${ }^{2}$, V. Werner $^{1}$, and $^{2}$ \\ M. Zweidinger ${ }^{1}$ \\ ${ }^{1}$ Institut für Kernphysik, Technische Universität Darmstadt, Schlossgartenstraße 9, 64289 Darmstadt, Germany \\ ${ }^{2}$ Institut für Angewandte Physik, Goethe-Universität Frankfurt, Max-von-Laue-Straße 1, 60438 Frankfurt a.M., Germany \\ ${ }^{3}$ ExtreMe Matter Institute EMMI and Research Division, GSI Helmholtzzentrum für Schwerionenforschung, Planckstraße 1, 64291 \\ Darmstadt, Germany \\ ${ }^{4}$ Frankfurt Institute for Advanced Studies, Ruth-Moufang-Straße 1, 60438 Frankfurt a.M., Germany \\ ${ }^{5}$ School of Engineering, University of the West of Scotland, Paisley PA1 2BE, United Kingdom \\ ${ }^{6}$ SUPA, Scottish Universities Physics Alliance, Glasgow G12 8QQ, United Kingdom
}

\begin{abstract}
The method of relative self absorption is based on the technique of nuclear resonance fluorescence measurements. It allows for a model-independent determination of ground-state transition widths, natural level widths, and, consequently, of branching ratios to the ground state for individual excitations. Relative selfabsorption experiments have been performed on the nuclei ${ }^{6} \mathrm{Li}$ and ${ }^{140} \mathrm{Ce}$. In order to investigate the total level width for the $0_{1}^{+}, T=1$ level at $3563 \mathrm{keV}$ in ${ }^{6} \mathrm{Li}$, a high-precision self-absorption measurement has been performed. In the case of ${ }^{140} \mathrm{Ce}$, self absorption has been applied for the first time to study decay widths of dipole-excited states in the energy regime of the pygmy dipole resonance.
\end{abstract}

\section{Introduction}

The Relative Self-Absorption (RSA) [1,2] approach allows for the direct determination of ground-state transition widths $\Gamma_{0}$, of natural level widths $\Gamma$, and, as a consequence, also of branching ratios $\Gamma_{0} / \Gamma$ to the ground state of individual states in a model-independent way. Self-absorption experiments are based on Nuclear Resonance Fluorescence (NRF) [3], i.e., photon-scattering measurements. Due to the low angular momentum transfer of photons, they are ideally suited to study low-lying dipole excitations up to the neutron separation threshold.

Various topics can be addressed via the RSA technique. RSA can be exploited to measure life-times $\tau$ of excited states below $\tau \sim 10^{-12} \mathrm{~s}$. Being independent from any calibration standards, it allows, in turn, for an improvement of the accuracy of calibration standards that are, e.g., used in NRF measurements for the photon-flux calibration [4]. In the present work, the level width $\Gamma$ of the $J^{\pi}=0_{1}^{+}$state with isospin $T=1$ of ${ }^{6} \mathrm{Li}$ at an excitation energy of $3.56 \mathrm{MeV}$ has been addressed. This state is the first state in a light nucleus which predominantly decays via the emission of $\gamma$ rays. The decay to the $1^{+}$ ground state is of $M 1$ character and the total decay width was previously measured to $\Gamma=8.19(17) \mathrm{meV}$ [5]. Precise knowledge of this decay width serves as a test case for various models such as ab-initio calculations. For in-

ae-mail: romig@ikp.tu-darmstadt.de stance, recently, it has been demonstrated that chiral currents can contribute up to $10 \%$ to $M 1$ transition strengths [6]. However, for a sensitive test of these contributions, a measurement of the $M 1$ decay width of the $0_{1}^{+}$level of ${ }^{6} \mathrm{Li}$ to an accuracy of better than $1 \%$ is desirable.

Additionally, the excitation and the decay pattern of individual states can be investigated at the same time. Only recently, NRF measurements exploiting the quasi-monochromatic $\gamma$-ray beams provided by the High Intensity $\vec{\gamma}$-Ray Source $(\mathrm{HI} \vec{\gamma} \mathrm{S})$ [7] at Triangle Universities Nuclear Laboratory (TUNL) in Durham, NC, USA have been performed to investigate the average decay pattern of dipole-excited states in the energy regime of the Pygmy Dipole Resonance (PDR) [8-12]. The PDR is an accumulation of enhanced E1-transition strength in the vicinity of the neutron-separation threshold [13]. Although often related to an out-of-phase oscillation of excess neutrons against the isospin-saturated core, its nature is still under debate and its properties are not yet fully determined. The measurements at $\mathrm{HI} \vec{\gamma} \mathrm{S}$ revealed that on average the decay via intermediate states, i.e., not directly to the ground state, cannot be neglected. However, at present, the experimental picture for the individual levels is not clear. Consequently, a determination of $\Gamma_{0}$ and $\Gamma_{0} / \Gamma$ for individual states is highly desirable to provide new information on the decay pattern of the PDR. Therefore, a relative selfabsorption experiment on the semi-magic nucleus ${ }^{140} \mathrm{Ce}$ has been performed. 
The measurements on ${ }^{6} \mathrm{Li}$ and ${ }^{140} \mathrm{Ce}$ have been conducted at the Darmstadt High Intensity Photon Setup (DHIPS) [14]. An extensive description of the measurements and the analysis is provided in Ref. [2]. In the following, the method of RSA will be introduced shortly. Furthermore, the results achieved from both measurements will be presented and discussed.

\section{The RSA Method}

In RSA experiments, an absorption target (absorber) made of the material of interest is irradiated with a $\gamma$-ray beam and the modified photon flux having passed through the absorber is investigated. In particular, the resonant absorption of photons resulting in absorption lines in the spectral distribution is studied. The resonant-absorption effect is directly related to the ground-state transition width $\Gamma_{0}$. Absorption lines are more pronounced for excited states with large $\Gamma_{0}$ and, thus, a measure for this quantity. However, being mostly a few $\mathrm{eV}$ wide and being also affected by atomic-attenuation effects in the absorber, they cannot be measured directly exploiting high-resolution $\gamma$-ray spectroscopy. At this point, the idea of self absorption enters, suggesting to use a scattering target (scatterer) which is made of the same material as the absorber to quantify the resonant absorption in the absorber. For this purpose, commonly two measurements are performed, the actual RSA measurement where the $\gamma$-ray beam passes through the absorber before irradiating the scatterer and a standard NRF measurement where the scatterer is irradiated with an unmodified beam. In this scenario, the self absorption

$$
R=\frac{N_{\mathrm{NRF}}-N_{\mathrm{RSA}} / f}{N_{\mathrm{NRF}}}
$$

is defined as the relative decrease of the number of NRF reactions $N_{\mathrm{RSA}}$ in the scatterer in the RSA measurement with respect to the corresponding number of reactions $N_{\mathrm{NRF}}$ in the NRF measurement, i.e., the NRF measurement serves as a reference. However, the decrease of NRF reactions cannot solely be attributed to resonant absorption of photons. It is also affected by atomic attenuation effects. Those can be corrected for by measuring relative to a monitoring target (monitor) which, for this purpose, is added to the scatterer. Ideally, the monitor has a few strongly excited states which are well distributed over the regarded energy range. The transition widths of the monitor must not be known. The decrease of NRF reactions in the monitoring target is exclusively attributed to atomic attenuation effects. Consequently, with the number of scattering reactions $N_{\mathrm{NRF}}^{\mathrm{mon}}$ and $N_{\mathrm{RSA}}^{\mathrm{mon}}$ in the monitor, a correction factor $f=N_{\mathrm{RSA}}^{\mathrm{mon}} / N_{\mathrm{NRF}}^{\mathrm{mon}}$ can be determined which allows for the correction of the atomic-attenuation effect. Additionally, the monitor corrects for variations of measuring times, photon-flux intensities, dead times, and other possible global normalisation factors. Consequently, the systematic uncertainties are drastically reduced.

The experimentally determined self absorption has to be compared to an analytically deduced expression of the

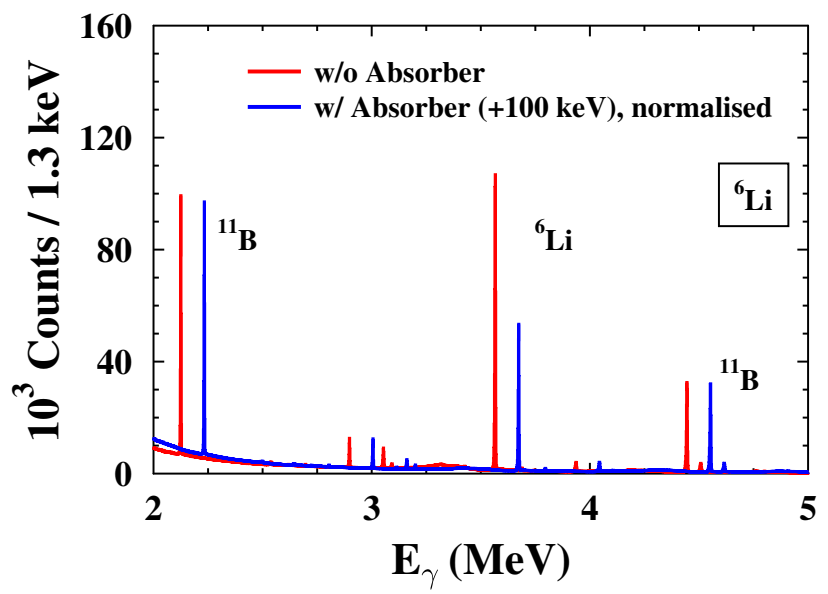

Figure 1. (Colour online.) ${ }^{6} \mathrm{Li}$ spectra recorded during the NRF (red) and the RSA (blue) measurement. The RSA spectrum is shifted by $100 \mathrm{keV}$ for the sake of clarity. It has been normalised, i.e., the correction factor $f$ has been applied to this spectrum.

self absorption $R_{\Gamma_{0}, \Gamma}$ depending on $\Gamma_{0}$ and $\Gamma$ to extract these quantities. It is obtained via

$$
R_{\Gamma_{0}, \Gamma}=\frac{\int_{-\infty}^{\infty} \int_{d_{A}}^{d_{A}+d_{S}} \alpha(E, z) \cdot d E d z}{\int_{-\infty}^{\infty} \int_{0}^{d_{S}} \alpha(E, z) \cdot d E d z}
$$

where the resonance-absorption density $\alpha(E, z)$ is integrated over the effective length of the scatterer. It represents a measure of the probability for resonant absorption of a photon with energy $E$ at a penetration depth $z$ into the target. The quantities $d_{A}$ and $d_{S}$ denote the thickness of the absorber and the scatterer.

From a comparison of $R$ and $R_{\Gamma_{0}, \Gamma}$, the ground-state transition width $\Gamma_{0}$ can be extracted. In combination with the result for $\Gamma_{0}^{2} / \Gamma$ from the NRF experiment, the natural level width $\Gamma$ and subsequently the branching ratio $\Gamma_{0} / \Gamma$ to the ground state can be deduced, as well. During the analysis, the so-called Bayesian approach [15] and the principle of maximal information entropy [16] have been exploited. In this approach, probability-density functions of all quantities have consequently been used to propagate the uncertainties in the measurement. This approach allows also for the exclusion of non-physical parts of these distributions such as $R<0$ or $\Gamma_{0} / \Gamma>1$. The results presented in the following are the most probable values of these probabilitydensity functions together with the $68.3 \%$ confidence interval surrounding it. However, these numbers should not be misinterpreted as classical Gaussian uncertainties.

\section{High-Precision RSA Measurement on ${ }^{6} \mathrm{Li}$}

Figure 1 shows the spectra that have been taken during the NRF and the RSA measurement on ${ }^{6} \mathrm{Li}$. The RSA spectrum has been normalised to the NRF spectrum exploiting a ${ }^{11} \mathrm{~B}$ monitoring target. Therefore, the peaks corresponding to transitions of ${ }^{11} \mathrm{~B}$ are equally high. The deviation between the measured intensities corresponding to the ground-state transition of the $0_{1}^{+}$state of ${ }^{6} \mathrm{Li}$ can be 


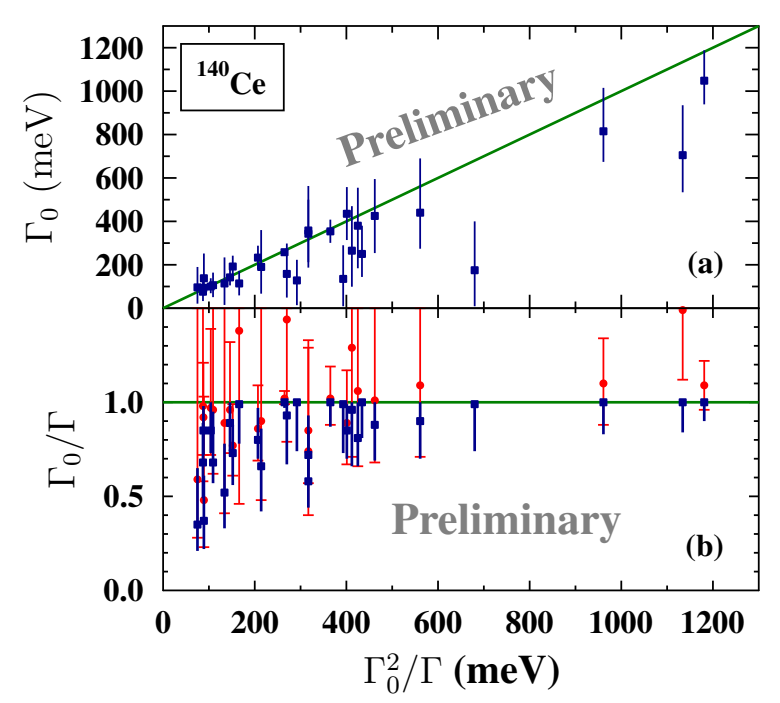

Figure 2. (Colour online.) (a) Ground-state transition widths and (b) the corresponding branching ratios to the ground state for 29 excited states of ${ }^{140} \mathrm{Ce}$ as determined from the RSA measurement. The blue squares represent the results of the Bayesian analysis approach, whereas the red circles correspond to a conventional analysis. The green lines mark $\Gamma_{0}=\Gamma$.

exclusively attributed to resonant absorption. The self absorption of this level accounts to $R=0.5193(15)$ corresponding to a relative uncertainty of only $0.3 \%$. For the measurements, $\mathrm{Li}_{2} \mathrm{CO}_{3}$ has been used as target material. However, the Debye temperature, a crucial ingredient of the analysis of RSA measurements, of ${ }^{6} \mathrm{Li}$ in this chemical compound is not yet measured. Consequently, the corresponding $M 1$ decay width cannot yet be determined. Nevertheless, independent of the actual Debye temperature the relative uncertainty of the decay width accounts to $\sim 1.1 \%$ which demonstrates the high precision achievable with the RSA approach.

\section{Decay Pattern of the PDR in ${ }^{140} \mathrm{Ce}$}

From the RSA experiment on ${ }^{140} \mathrm{Ce}$, ground-state transition strengths as well as branching ratios to the ground state have been determined for 29 excited states in the energy regime of the PDR. The corresponding results are shown in Fig. 2 as a function of $\Gamma_{0}^{2} / \Gamma$, which is the result of the NRF measurement on ${ }^{140} \mathrm{Ce}$. Figure 2 (a) depicts the ground-state transition widths $\Gamma_{0}$. The green line corresponds to $\Gamma_{0}=\Gamma_{0}^{2} / \Gamma$, i.e., $\Gamma_{0} / \Gamma=1$. In most cases, the results for $\Gamma_{0}$ agree with this line within their $68.3 \%$ confidence interval. Thus, they predominantly decay directly to the ground state. However, in particular the weaker excited states exhibit also values of $\Gamma_{0}$ that are larger than $\Gamma_{0}^{2} / \Gamma$ which means that they have a branching ratio to the ground state being smaller than one. Eventually, in a few cases, $\Gamma_{0}$ has been found to be significantly smaller than $\Gamma_{0}^{2} / \Gamma$ which would correspond to a non-physical branching ratio to the ground state being larger than one. Those cases may be explained when an observed peak in the measured spectra corresponds to two levels being located within such a close proximity to each other that they cannot be resolved with the given experimental resolution. In such a case, the self absorption of the supposedly single state with combined photon scattering cross section $\propto \Gamma_{0}^{\prime 2} / \Gamma^{\prime}=\sum \Gamma_{0, i}^{2} / \Gamma_{i}$ would be overestimated as compared to the true self absorption values due to the individual widths $\Gamma_{0, i}<\Gamma_{0}^{\prime}$ resulting in a too large or even non-physical value for the would-be branching ratio $\Gamma_{0}^{\prime} / \Gamma^{\prime}$.

The corresponding ground-state branching ratios $\Gamma_{0} / \Gamma$ are shown in Fig. 2 (b). The red circles correspond to a conventional analysis of the RSA experiment, whereas the blue squares illustrate the results of the Bayesian analysis approach. With this approach, the non-physical part $\Gamma_{0} / \Gamma>1$ has been excluded. As a consequence, branching ratios being larger than one are shifted to the physically allowed region. The obtained data for $\Gamma_{0} / \Gamma$ show that the rather strongly excited states decay predominantly directly back to the ground state. In contrast, more weakly excited states exhibit smaller branching ratios to the ground state and, hence, also decay via intermediate states.

These observations provide a first detailed insight in the decay pattern of the pygmy dipole resonance and may be used for a comprehensive test of the nuclear statistical model in the energy regime of the PDR.

\section{Acknowledgements}

The authors would like to thank the operating team of the S-DALINAC as well as all scientists on shift during the measurements. This work was supported by the Deutsche Forschungsgemeinschaft (Contract No. SFB 634 and SO907/2-1), by the Alliance Program of the Helmholtz Association (HA216/EMMI), and by HIC for FAIR within LOEWE launched by the state of Hesse, Germany.

\section{References}

[1] R. Vodhanel et al., Phys. Rev. C 29, 409 (1984)

[2] C. Romig, Ph.D. thesis, TU Darmstadt (2014)

[3] U. Kneissl, H. Pitz, A. Zilges, Prog. Part. Nucl. Phys. 37, 349 (1996)

[4] N. Pietralla et al., Phys. Rev. C 51, 1021 (1995)

[5] D.R. Tilley et al., Nuclear Physics A 708, 3 (2002)

[6] S. Pastore et al., Phys. Rev. C 87, 035503 (2013)

[7] H.R. Weller et al., Prog. Part. Nucl. Phys. 62, 257 (2009)

[8] A.P. Tonchev et al., Phys. Rev. Lett. 104, 072501 (2010)

[9] C.T. Angell et al., Phys. Rev. C 86, 051302 (2012)

[10] J. Isaak et al., Physics Letters B 727, 361 (2013)

[11] M. Scheck et al., Phys. Rev. C 87, 051304(R) (2013)

[12] C. Romig et al., Phys. Rev. C 88, 044331 (2013)

[13] D. Savran, T. Aumann, A. Zilges, Prog. Part. Nucl. Phys. 70, 210 (2013)

[14] K. Sonnabend et al., Nucl. Instr. and Meth. A 640, 6 (2011)

[15] K. Weise et al., Rad. Prot. Dos. 121, 52 (2006)

[16] E. Jaynes, Phys. Rev. 106, 620 (1957) 
